\title{
MAGIC2-D Simulations of High Efficiency Klystrons using the Core Oscillation Method
}

\author{
David A. Constable, Chris Lingwood, Graeme Burt \\ Engineering Department, Lancaster University \\ Lancaster, United Kingdom \\ d.constable@lancaster.ac.uk
}

\author{
Andrey Yu. Baikov \\ Moscow University of Finance \& Law \\ Moscow, Russia
}

\begin{abstract}
Klystrons employing traditional monotonic electron bunching are capable of efficiencies up to $\sim 70 \%$. The use of the core oscillation method (COM) of electron bunching has predicted a significant improvement in efficiency towards $90 \%$. Here, we document refinements on previously presented geometries, with PIC simulations predicting efficiencies up to $85 \%$.
\end{abstract}

Keywords—klystron; high efficiency; core oscillation method; MAGIC2-D.

\section{INTRODUCTION}

Several large-scale particle accelerators projects, such as the Compact Linear Collider (CLIC) [1] and the Future Circular Collider (FCC) [2] are currently in the design stage. Their RF requirements are expected to be on the order of $100 \mathrm{MW}$. Due to the significant increase in RF power demand when compared to the currently operating Large Hadron Collider (LHC) [3], the efficiency of the RF sources should be maximized, to reduce the overall operational and installation costs.

Klystrons are the most common multi-MW RF power sources which are used to drive particle accelerators. The existing commercialized klystron technology offers efficiencies up to $70 \%$ in such devices. Recent studies in novel klystron bunching mechanisms [4], including the core oscillation (COM) $[5,6]$ and BAC [6-8] methods have predicted increases in efficiencies to greater than $80 \%$.

PIC simulations of an $82 \%$ efficient single beam COM klystron has been reported [9]. In this paper, we present subsequent refinements which result in an increase in predicted efficiency up to $85 \%$.

\section{NUMERICAL SIMULATION}

The original GSP-08_04 klystron is an 8 cavity, $800 \mathrm{MHz}$, 1.4 MW tube with an electron beam voltage of $133.85 \mathrm{kV}$, beam current of $12.551 \mathrm{~A}$, and simulated efficiency of $82 \%$ [9]. This klystron design was used as a starting point for refined postoptimization in an attempt to obtain improved performance. This optimization was done using the 1D klystron code KlypWin [5] which allows for global optimization with many (up to 50) free parameters. Through optimization, the drift tube and electron

\author{
Igor Syratchev \\ CERN \\ Geneva, Switzerland
}

\author{
Richard Kowalcyzk \\ SLAC National Accelerator Laboratory \\ Menlo Park, California, United States
}

beam radii, beam voltage and current, cavities gaps and impedances were kept constant, whist all the other parameters were set free within a few percent window. In the process over 20,000 cases were simulated, of which 10 tubes were selected for further analysis using the 1D code AJDISK [10] and the 2D PIC code MAGIC [11]. The performances of these tubes simulated by the various codes are summarized in Figure 1. All tubes showed good performance in the wide range of parameters, with similar trends over the different codes being used. For further illustration, we have selected the tube which showed best performance with $84.64 \%$ efficiency obtained in 2D PIC simulations (referred as $08 \_04$ 08).

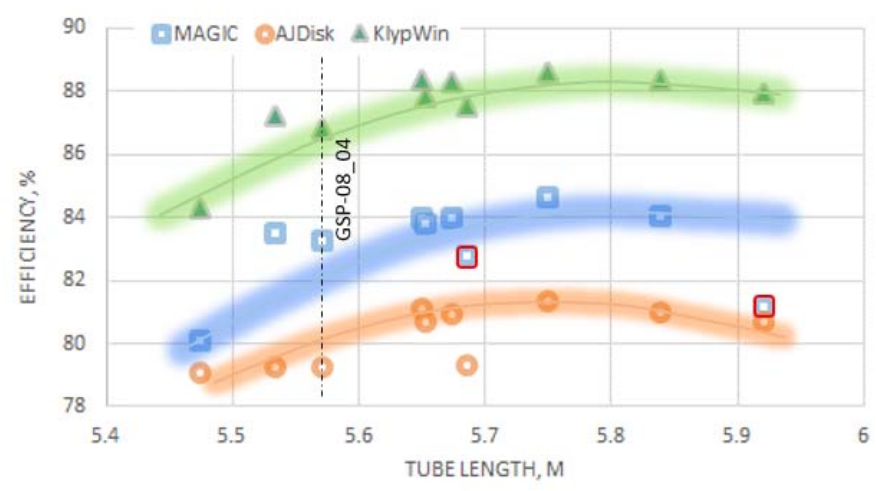

Figure 1 - Predicted efficiencies of 10 selected klystrons simulated by different computer codes.

On simulating this tube in MAGIC2-D, a maximum output power of $1.42 \mathrm{MW}$ in saturation was achieved for an input power of $6 \mathrm{~W}$, corresponding to power gain of $53.7 \mathrm{~dB}$. For all input power levels considered, the output power is stable, with no reflected electrons predicted. The instantaneous bunch profiles in $\mathrm{P}_{\mathrm{Z}} \mathrm{Z}$ and $\mathrm{R}-\mathrm{Z}$ phase-spaces at the final two cavities are shown in Figure 2. These simulations confirm that COM is a reliable method to provide strong bunch saturation. The number of electrons contained in the anti-bunch (Figure 3 ) is less than $1 \%$ compared with the bunch.

The radial bunch stratification, i.e. radial variation of the bunch length at the output cavity entrance is the common issue 
in klystrons that limits their performance. This effect is due to the radial mismatch between space charge forces and kinetic momentum received by electrons from bunching cavities. As a result, the longitudinal modulation depth is proportional to the radial offset within the bunch. Ultimately, the particles located close to the bunch radial edge will receive excessive deceleration in the output cavity and will be reflected. This effect was partially mitigated in the 08 _04 and 08_04_08 klystron designs by tuning of the last three bunching cavities. These cavities mostly affect the electrons at the bunch longitudinal periphery with least effect on the bunch core. However, the bunch still has visible radial stratification. This can be illustrated further in Figure 4, where Applegate diagrams calculated at different radial offsets are shown.
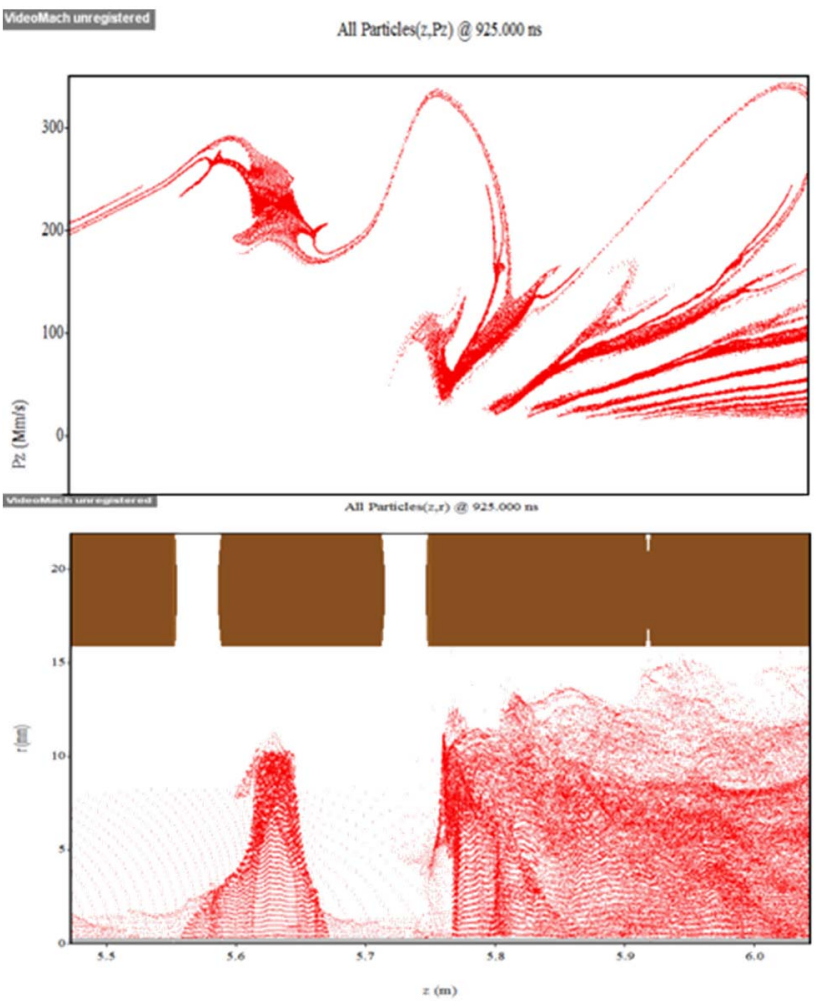

Figure $2-\mathbf{P}_{\mathrm{Z}} \mathrm{Z}$ (top) and RZ (bottom) phase-space profiles of the electron beam at the final two cavities.

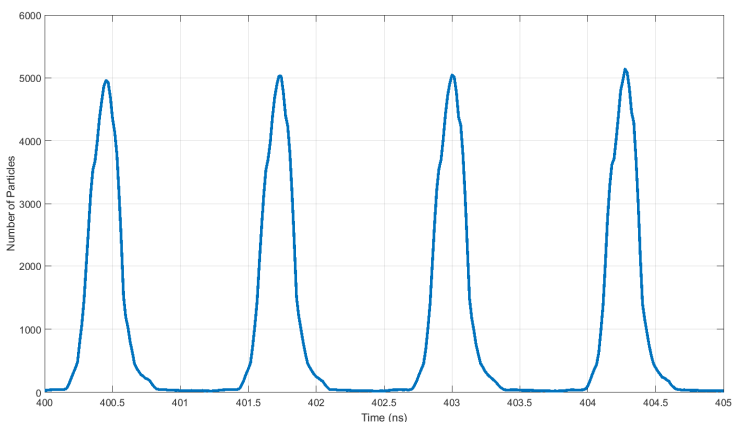

Figure 3 - Bunch time profiles integrated locally at the entrance of the output cavity.

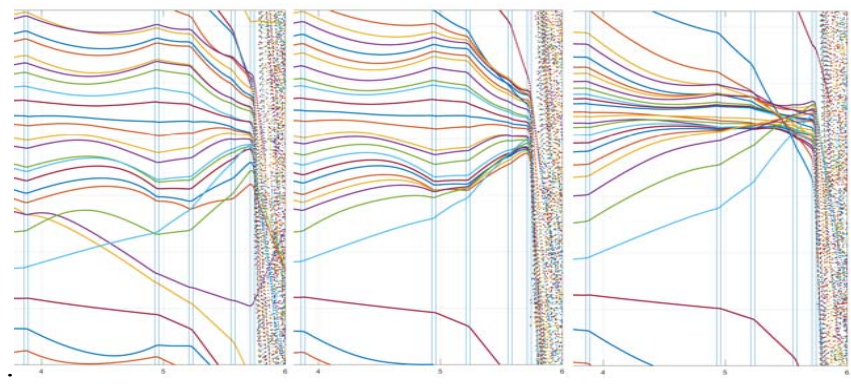

Figure 2-Applegate diagrams for on-axis particles (left), $\mathbf{x} 0.5 \mathrm{R}$ beam (center) and close to $\mathrm{R}$ beam (right) in MAGIC2-D.

\section{SUMMARY}

Post optimizations of the GSP 08_04 klystron have been performed. In the new design, the efficiency is increased from original $82.7 \%$ up to $84.64 \%$. This enhancement of $2 \%$ is incredibly important when considering the impact on the RF demands of future particle accelerators.

\section{ACKNOWLEDGMENTS}

The authors would like to thank all the members of the High Efficiency International Klystron Activity (HEIKA). They would also like to thank ATK's Alliance Partnership Program for the use of MAGIC2-D.

\section{REFERENCES}

[1] M. Aicheler, P. Burrows, M. Draper, T. Garvey, et al., "A Multi-TeV linear collider based on CLIC technology: CLIC Conceptual Design Report," CERN-2012-007, 2012.

[2] M. Koratzinos, "FCC-ee accelerator parameters, performance and limitations," 37th Conference on High Energy Physics (ICHEP), Valencia, Spain, 2014.

[3] E. Ciapala, L. Arnaudon, P. Baudrenghien O. Brunner, et al., "Commisioning of the $400 \mathrm{MHz}$ LHC RF system," $11_{\text {th }}$ European Particle Accelerator Conference (EPAC), Genoa, Italy, 2008.

[4] D. A. Constable, G. Burt, et al., "High efficiency klystron development for particle accelerators", in Proc. EE-FACT'16, Daresbury, United Kingdom, paper WET3AH2.

[5] A. Yu. Baikov, O. A. Grushina, and M. N. Strikhanov, "Simulations of conditions for the maximial efficiency of decimeter-wave klystrons", Technical Physics, 59(3), pp. 421-427, 2014.

[6] A. Yu. Baikov, C. Marrelli, and I. Syratchev, "Toward high-power klystrons with RF power conversion efficiencies on the order of $90 \%$ ", IEEE. Trans. Elec. Dev., 62(10), pp. 3406-3412, 2015.

[7] A. Jensen et al., "Increasing klystron efficiency using COM and BAC tuning and application to the 5045 klystron", in Proc. 2016 Int. Vacuum Electronics Conference. (IVEC‘16), Monterey, USA, April 2016.

[8] I. A. Guzilov, "BAC method of increasing the efficiency in klystrons", in Proc. 10 th Int. Vacuum Elec. Sources Conf. (IVESC '14), Saint Petersburg, Russia, July 2014.

[9] D. A. Constable, C. Lingwood, G. Burt, et al., "2-D Particle-in-Cell simulations of high efficieicny klystrons", in Proc. 2016 IEEE International Vacuum Electronics Conference (IVEC'16), Monterey, USA, April 2016.

[10] A. J. Jensen et al., "Developing sheet beam klystron simulation capability in AJDISK", IEEE Trans. Elec. Dev., vol. 61(6), pp. 1666-1671, Jan. 2014

[11] B. Goplen et al, "User-configurable MAGIC for electromagnetic PIC calculations," Computer Physics Communications, vol. 87, pp. 54-86, May 1995. 